\title{
High frequency immittance for neonates: a normative study
}

De Wet Swanepoel; Sonia Werner; René Hugo; Brenda Louw; Rina Owen; André Swanepoel

Department of Communication Pathology, University of Pretoria, South Africa

\section{Abstract}

Conclusion. High frequency immittance measurements demonstrate promise in clarifying middle ear status for neonates but age- and gender-specific norms should be consulted. Objective. To describe high frequency immittance measurements using a $1000 \mathrm{~Hz}$ probe tone for a sample of 278 neonatal ears (0-4 weeks of age) in order to compile normative tympanometric and acoustic reflex criteria. Subjects and methods. Assessment of neonatal ears included $1000 \mathrm{~Hz}$ probe tone immittance measurements (tympanograms and ipsilateral acoustic reflexes), and distortion product oto-acoustic emission (DPOAE) screening. Results were compared and normative values were compiled for immittance measures in ears controlled for normal middle ear functioning $(n=250)$. Results.

Comparison of immittance results to OAE screening outcome provides estimates of sensitivity and specificity for middle ear fluid with tympanometry of $57 \%$ and $95 \%$, and $57 \%$ and $90 \%$ for acoustic reflex presence, and $58 \%$ and $87 \%$ for combined tympanogram and acoustic reflex results, respectively. Normative data indicate that static peak admittance values differ significantly across gender and age with the 5th percentile cut-off value for the entire sample at 1.4 mmho. The $90 \%$ range of tympanic peak pressure normative values increases with increasing age from $140 \mathrm{daPa}$ for neonates 1 week of age to $210 \mathrm{daPa}$ for neonates 2-4 weeks of age. Acoustic reflexes were elicited at $93 \pm 9 \mathrm{~dB}$ with a $90 \%$ normality range of $80-105 \mathrm{~dB}$.

Keywords: acoustic reflex; high frequency probe tone; immittance; middle ear fluid; middle ear effusion; neonatal hearing screening; static peak admittance; tympanic peak pressure; tympanometry

\section{Introduction}

As newborn and infant hearing screening programmes are being implemented as the standard of care in a number of world regions the need for an accurate measure to differentiate between true positive and false positive results due to middle ear fluid is becoming increasingly important. Newborns and infants with mild conductive hearing loss often refer hearing screenings resulting in higher false positive rates that lead to added expenses, decreased programme efficacy, and increased chances of anxiety for parents ${ }^{1,2}$. Even though significant efforts have been made to reduce the number of false positive results by comprehensive screening protocols, the question of compromised middle ear functioning due to otitis media with effusion, a transient middle ear effusion, 
or middle ear cavities containing mesenchyme and not fully pneumatized at birth, has not yet been addressed sufficiently ${ }^{3}$. Middle ear fluid or middle ear effusion is a common diagnosis among newborns and infants, with infants in neonatal intensive care units (NICUs) especially prone to the condition ${ }^{4,5}$. A recent study has pointed out that otitis media with effusion may contribute up to $67 \%$ of the false positive newborn hearing screens ${ }^{5}$. The presence of middle ear fluid severely diminishes and even obliterates otoacoustic emissions (OAEs) and, while auditory brainstem response (ABR) screening is more robust with fewer failures due to mild middle ear conduction pathologies, it may still lead to a refer result in more serious cases ${ }^{6-10}$.

Unfortunately it is difficult to diagnose middle ear fluid in the newborn and young infant after referral on a screening test. The effect of sound transmission through the neonatal ear canal to the middle ear and cochlea has not been described sufficiently and is therefore not clearly understood ${ }^{3}$. Conventional immittance evaluations of middle ear functioning with a $226 \mathrm{~Hz}$ probe tone have been demonstrated to be unreliable for accurate assessment of middle ear status in infants younger than 7 months of age ${ }^{11-13}$. Reports have indicated that the use of low frequency probe tones to record tympanograms leads to a high false negative rate, which means a poor sensitivity rate for detecting middle ear pathology ${ }^{12,14}$. The sharp sensitivity contrast of $226 \mathrm{~Hz}$ tympanometry in young infants and adults has been attributed to the fact that the young infant's middle ear is mass-dominated compared with a stiffness-dominated system in adults. Low frequency probe tones are well suited to evaluate stiffness-dominated systems and not the massdominated middle ears of young infants ${ }^{11,15}$.

The use of high frequency probe tones has been suggested as a more reliable means of assessing a middle ear system which is mass-dominated as in the case of young infants. As a result a number of clinical programmes in several countries have included high frequency tympanometry as part of the screening protocol. Recent reports have demonstrated promising preliminary results using a $1000 \mathrm{~Hz}$ probe tone to detect middle ear dysfunction in neonates ${ }^{14,16-18}$. Some normative guidelines based on limited samples of neonates have been suggested by Margolis et al. ${ }^{17}$ and Kei et al. ${ }^{16}$. Margolis et al. ${ }^{17}$ suggested that a single cut-off value for static admittance of $0.6 \mathrm{mmho}$ is useful for infants up to 4 weeks chronological age. The study by Kei et al. ${ }^{16}$ classified $1000 \mathrm{~Hz}$ tympanogram shapes in a group of neonates who passed their OAE screening. Three distinct shapes were identified including a peaked, double peaked and flat sloping tympanogram, while $0.8 \%$ of the tympanograms did not fit any of these shapes. Flat sloping shaped tympanograms, measured in $5.7 \%$ of cases, demonstrated less robust OAE responses, which may indicate a degree of compromised middle ear functioning too low to lead to an OAE refer but enough to result in no tympanic peak ${ }^{16}$.

An alternative technique suggested for possible neonatal middle ear assessment has been the acoustical admittance and reflectance (YR) response ${ }^{3}$. Initial results have indicated promise that the YR response is sensitive to both forward and reverse sound transmission through the ear canal and middle ear ${ }^{3}$. Unfortunately the complex nature of the response, with over 30 variables in the response itself, and limited normative data and clinical 
systems have prevented its use as a clinical tool for neonatal middle ear evaluation thus far.

A reliable test that is able to distinguish between sensorineural hearing loss and middle ear pathology for neonates and young infants will be an important way to identify screening failures caused by middle ear pathology to direct appropriate medical referral and diagnostic audiological evaluations ${ }^{17}$. The preliminary guidelines for interpreting $1000 \mathrm{~Hz}$ tympanometry hold significant promise for accountable application of the technique in neonatal screening programmes but require further normative studies on significant numbers of subject samples. This demand for more normative data is becoming increasingly important with the implementation of widespread UNHS programmes in developed and developing countries ${ }^{16-19}$. This study therefore investigated middle ear functioning in a sample of neonatal ears in order to describe normative data for high frequency immittance measurements using a $1000 \mathrm{~Hz}$ probe tone.

\section{Subjects and methods}

The institutional review board at the University of Pretoria approved this project before any data were collected to ensure that the procedures followed were in accordance with the ethical standards as specified by the Helsinki Declaration of 1975, as revised in 1983.

\section{Subjects}

Recordings were made from a total of 278 healthy neonatal ears (52\% female) from 143 neonates attending two maternal and child health clinics in the Hammanskraal community in South Africa. All the neonates were full-term babies and their ages at time of testing varied between 1 and 28 days. Informed consent was obtained from all caregivers before any testing commenced. The testing protocol conducted on these ears included high frequency immittance measurements (tympanograms and acoustic reflexes) and a distortion product oto-acoustic emission (DPOAE) screening. The test procedures were conducted on both ears for all neonates as far as possible but in certain cases only one ear could be evaluated or only one test conducted for an ear due to the infant's restlessness or irritability.

\section{Apparatus}

A GSI Tympstar (version 2) Middle-Ear Analyzer was used to record immittance measures. A high frequency probe tone of $1000 \mathrm{~Hz}$ was utilized to measure $\mathrm{Y}$-admittance tympanograms, with a positive to negative pressure sweep of $200 \mathrm{daPa}$ at a pump speed of $200 \mathrm{daPa} / \mathrm{s}$, as recommended for young infants ${ }^{11}$. The maximum point on a recorded tympanogram was marked to obtain the uncompensated peak admittance value with the corresponding pressure value at this point.

Ipsilateral reflexes at $1000 \mathrm{~Hz}$ using a $1000 \mathrm{~Hz}$ probe tone were also recorded with the GSI Tympstar. Reflexes were determined at the lowest intensity eliciting a reflex 
response with a deviation $>0.02$. This deviation was required to be repeatable and indicative of growth at higher intensities and decrease in amplitude at lower intensities.

The GSI AUDIOscreener was used for DPOAE screening. The test parameters used were according to the default screening protocol, setting 'DPOAE 2'. Five frequencies (2000, 3000, 4000, 5000 and $6000 \mathrm{~Hz}$ ) were assessed for each ear and a pass criterion was based on passing at least four of the five frequencies evaluated. The recording of DPOAE was based on a F2 centre method and the frequencies were measured in a downward order starting with the highest and ending at the lowest using a linear averaging method of analysis. The minimum signal-to-noise difference was set at $6 \mathrm{~dB}$ for all frequencies.

\section{Procedure}

Testing was conducted by three qualified audiologists. To avoid any order effects the immittance and OAE tests were conducted in a randomized manner on each neonate. This meant that some neonates were screened with OAE first followed by immittance testing and others were tested with immittance measures first followed by OAE screening. All testing was conducted in a quiet room dedicated to hearing screening. The neonate was placed in a comfortable position depending on state of wakefulness (in mother's arms or in a sleeping cot) before testing commenced.

To conduct immittance measures the infant's ear was investigated to select an appropriately sized probe tip. Once a good seal was attained $1000 \mathrm{~Hz}$ probe tone tympanograms were recorded. The peak of every tympanogram was marked with a cursor, thus providing the peak pressure ( $\mathrm{daPa}$ ) and peak compliance reading (mmho). If no peak was present it was recorded as such. The tympanograms were followed by the recording of acoustic reflexes with a $1000 \mathrm{~Hz}$ probe tone at a test frequency of $1000 \mathrm{~Hz}$. Measurements were initiated at $70 \mathrm{~dB} \mathrm{HL}$ and a reflex threshold seeking procedure of 10 $\mathrm{dB}$ up and $5 \mathrm{~dB}$ down increments was employed. A maximum intensity of $110 \mathrm{~dB}$ was tested. OAE screening was also conducted by investigating the ear canal for selection of an appropriate sized probe tip. Once a complete seal was attained the ear was screened. If the screen result referred the subject a second screening was conducted after readjusting and fitting the probe. If the second screen also yielded a refer result it was recorded as such and a follow-up appointment was scheduled.

The data recorded from these neonates were used to compile normative guidelines for high frequency immittance in neonates with normal middle ear functioning. To establish normative data for this group only the immittance data from neonatal ears with an OAE pass result and a discernible tympanogram peak were used ( $n=250 ; 52 \%$ female). An OAE pass result was not used as the only criterion because previous reports have indicated that in a small number of cases with middle ear fluid a pass result may still be obtained with OAE ${ }^{16,20}$. To assure that reliable norms are specified, as far as the performed procedures accuracy allows, a second criterion requiring a discernible tympanogram peak for inclusion was set. A recent report indicated that an OAE pass result was obtained in $5.7 \%$ of ears with no discernible tympanometric peak, although reduced OAE amplitudes were observed in these ears ${ }^{16}$. Since such cases may indicate a 
degree of compromised middle ear functioning and a discernible tympanometric peak is generally accepted as indicative of normal middle ear functioning with $1000 \mathrm{~Hz}$ tympanometry for neonates, this criterion was specified alongside an OAE pass result to ensure a high degree of confidence that only ears with normal middle ear functioning were included ${ }^{16,18}$.

\section{Results}

Of the 278 neonatal ears evaluated by tympanometry and OAE screening, 8\% (22/278) presented with no tympanometric peak and 5\% (14/278) did not pass the initial OAE screening. Absent acoustic reflexes were observed in 12\% (33/267) of the sample. No significant gender effect was found for any of these test procedures. Tympanograms with a double peaked configuration comprised 6\% (16/264) of the peaked tympanogram sample and 93\% (15/16) of these cases coincided with an OAE pass result. Even though this was a small subsample a significantly increased number of males (69\%) presented a double peaked tympanogram configuration.

The incidence of OAE pass results, peaked high frequency tympanograms and present acoustic reflexes for the sample of neonatal ears was $95 \%, 92 \%$ and $88 \%$, respectively. A comparison between the negative and positive predictive values of these test procedures as compared against each other is provided in Table I.

\section{Positive and negative predictive values for OAE, Table I. tympanogram (TYMPS) and acoustic reflex (AR) results for neonates. \\ TYMPS}

\begin{tabular}{llcll} 
Predictive measure & Peak $(\boldsymbol{n}=\mathbf{2 5 6})$ & $\begin{array}{c}\text { No peak } \\
(\boldsymbol{n}=\mathbf{2 2})\end{array}$ & $\begin{array}{r}\text { Present } \\
(\boldsymbol{n}=\mathbf{2 3 6})\end{array}$ & Absent $(\boldsymbol{n}=\mathbf{3 3})$ \\
OAE pass & $95 \%$ & $5 \%$ & $90 \%$ & $10 \%$ \\
OAE refer & $43 \%$ & $57 \%$ & $43 \%$ & $57 \%$ \\
Predictive measure & \multicolumn{2}{c}{ OAE } & \multicolumn{2}{c}{ AR }
\end{tabular}

$\begin{array}{lllll} & \text { Pass }(n=264) & \text { Refer }(n=14) & \text { Present }(n=234) & \text { Absent }(n=33) \\ \text { Tympanogram peak } & 98 \% & 2 \% & 93 \% & 7 \% \\ \text { Tympanogram no peak } & 64 \% & 36 \% & 32 \% & 68 \% \\ & & \text { OAE } & & \text { TYMPS }\end{array}$

\begin{tabular}{|c|c|c|}
\hline AR present & $98 \%$ & $3 \%$ \\
\hline AR absent & $76 \%$ & $24 \%$ \\
\hline
\end{tabular}

Measured against the OAE result the sensitivity and specificity for middle ear pathology with high frequency tympanometry, i.e. no discernible tympanometric peak indicating 
pathology, was $57 \%$ and $95 \%$, respectively. OAE results compared to acoustic reflex presence indicate a sensitivity and specificity value of $57 \%$ and $90 \%$, respectively, for high frequency probe tone acoustic reflex measurements in neonates.

\section{Normative data}

To establish reliable norms for $1000 \mathrm{~Hz}$ immittance two criteria were set for including ears from the current sample: (1) an OAE pass result and (2) a discernible tympanometric peak. These criteria reduced the sample size to 250 neonatal ears that were used to compile a normative set of data. Table II indicates the descriptive peak admittance and tympanic peak pressure values for this normative sample.

\section{The $1000 \mathrm{~Hz}$ tympanometry norms for neonates. Table II.}

\begin{tabular}{|c|c|c|c|c|c|}
\hline \multicolumn{2}{|c|}{$\begin{array}{c}<1 \text { week of age ( } n=73 \\
\text { ears) }\end{array}$} & \multicolumn{2}{|c|}{$\begin{array}{l}\text { 1-4 weeks of age } \\
(n=177 \text { ears) }\end{array}$} & \multicolumn{2}{|c|}{$\begin{array}{c}\text { 0-4 weeks of age } \\
\text { ( } n=250 \text { ears) }\end{array}$} \\
\hline $\begin{array}{c}\text { Peak } \\
\text { admittance }\end{array}$ & $\begin{array}{c}\text { TPP } \\
\text { (daPa) }\end{array}$ & $\begin{array}{c}\text { Peak } \\
\text { admittance }\end{array}$ & $\begin{array}{c}\text { TPP } \\
\text { (daPa) }\end{array}$ & $\begin{array}{c}\text { Peak } \\
\text { admittance }\end{array}$ & $\begin{array}{c}\text { TPP } \\
\text { (daPa) }\end{array}$ \\
\hline 2.2 & -10 & 2.4 & 5 & 2.4 & -1 \\
\hline 0.9 & 48 & 0.7 & 49 & 0.8 & 49 \\
\hline 7.7 & 185 & 5.1 & 115 & 7.7 & 185 \\
\hline 1 & -130 & 1.2 & -185 & 1 & -185 \\
\hline 1.2 & -70 & 1.5 & -80 & 1.4 & -75 \\
\hline 2.0 & -10 & 2.3 & 5 & 2.2 & -5 \\
\hline 3.4 & 70 & 3.8 & 85 & 3.7 & 80 \\
\hline
\end{tabular}

Statistical analysis indicates that there is a significant difference ( $p<0.01$; $t$ test with pooled variance) between the peak admittance values for male and female ears. No statistically significant differences were obtained between the tympanic peak pressure values. Comparing different age groups within the sample of neonatal ears also indicated statistically significant differences for admittance values but not for tympanic peak pressure values. The admittance values for neonates younger than 1 week of age compared to those older than 1 week of age differed significantly on the $5 \%$ level of significance ( $p<0.05$; $t$ test with pooled variance). When the admittance values for neonates younger than 1 week of age were compared to those of neonates who were 4 weeks of age a significant difference on the $1 \%$ level of significance was evident $(p<0.01 ; t$ test with pooled variance). The general trend indicates significantly increasing admittance values with increasing age in the neonatal period. These differences necessitate the consideration of age- and gender-specific normative data for the use of $1000 \mathrm{~Hz}$ probe tone tympanometry in neonates. Figures 1 and 2 provide a detailed presentation of admittance and tympanic peak pressure values for neonatal ears according to gender and age. 


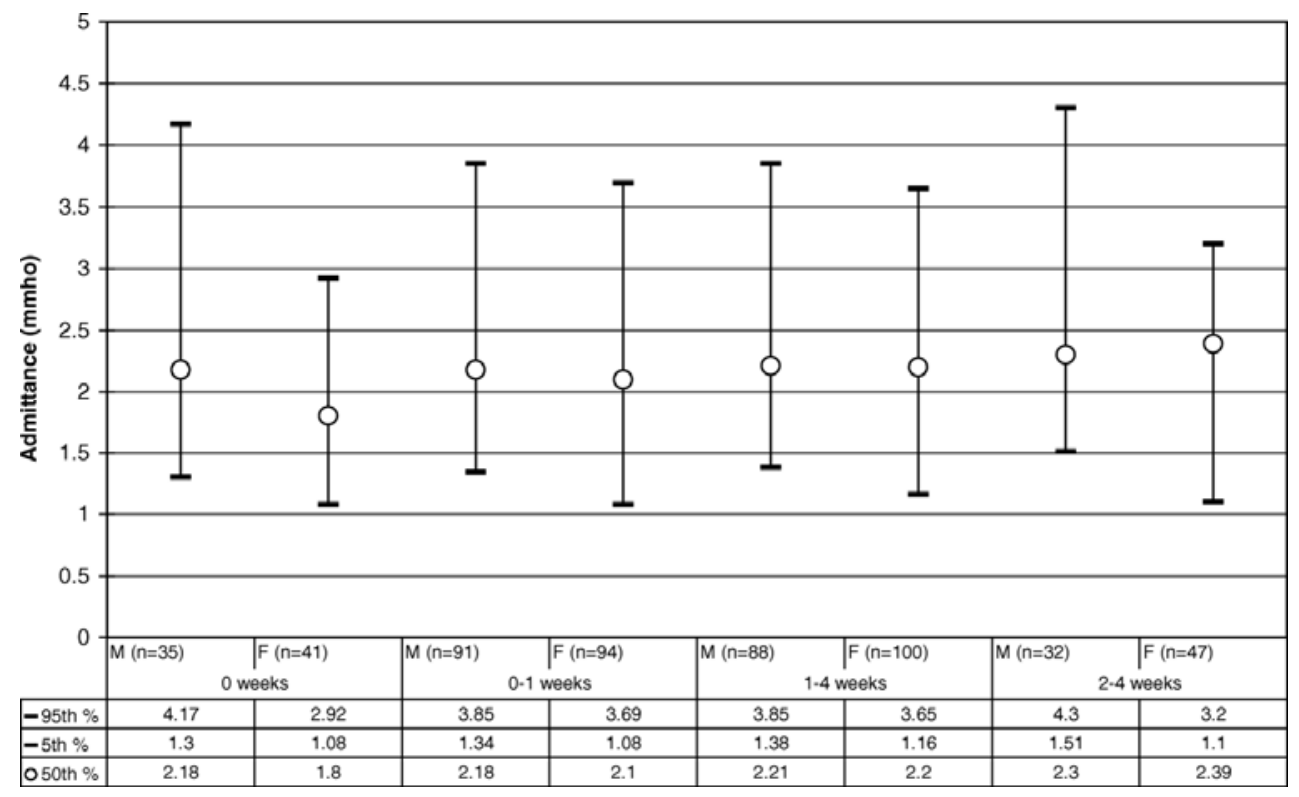

Figure 1. Age- and gender-specific admittance norms for neonates.

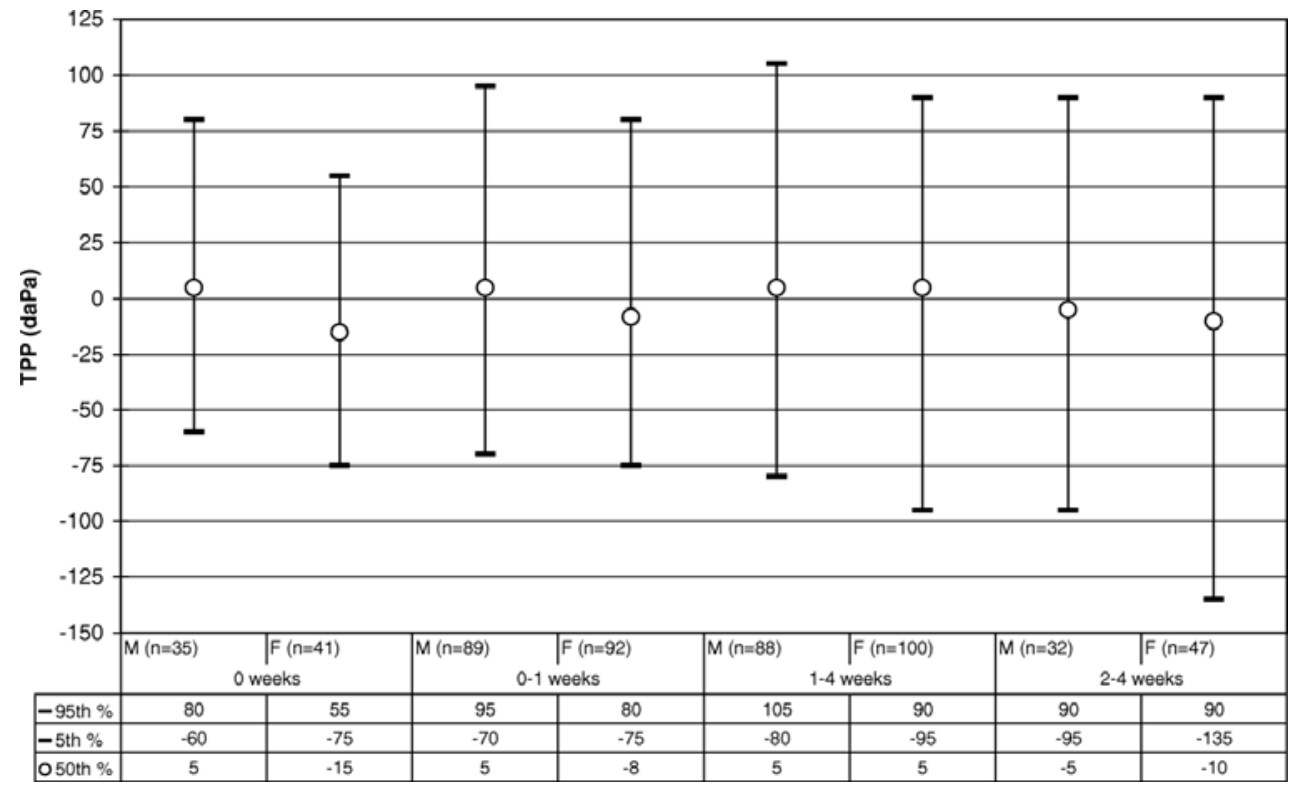

Figure 2. Age- and gender-specific tympanic peak pressure norms for neonates.

The range of admittance values across age groups for the lower limit (5th percentile) was between 1.30 and 1.51 mmho for male ears and between 1.08 and 1.16 mmho for female ears. The range of admittance values across age groups for the upper limit (95th percentile) was between 3.85 and 4.30 mmho for male ears and between 2.92 and 3.69 mmho for female ears. The median values for the male ears across all age groups varied between 2.18 and 2.30 mmho and for the female ears across all age groups between 1.80 and 2.39 mmho. 
The range of tympanic peak pressure values for the lower limit (5th percentile) across age groups varied between -95 and -60 daPa for male ears and between -135 and -75 daPa for female ears. The range of tympanic peak pressure values for the upper limit (95th percentile) across age groups varied between 80 and 105 daPa for male ears and between 55 and $90 \mathrm{daPa}$ for female ears. Median values varied between -15 and $5 \mathrm{daPa}$ across gender and age groups.

In all age groups the female ears had lower or equivalent 5th and 95th percentile values. The values across age groups also indicated the smallest $90 \%$ tympanic peak pressure range (5th to 95th percentile) for male (140 daPa) and female ears (130 daPa) younger than 1 week of age. The highest $90 \%$ tympanic peak pressure value range was obtained in the oldest neonatal ears (185 daPa) for both male and female ears. The tympanic peak pressure 90 th percentile range therefore increases with increasing age across gender.

\section{Acoustic reflex thresholds for neonates}

Acoustic reflexes were present in $94 \%$ of ears with peaked tympanograms. No significant differences were obtained across gender or age for acoustic reflex thresholds in neonates. The mean threshold was $93 \mathrm{~dB} \pm 9 \mathrm{~dB}$ with a maximum of $110 \mathrm{~dB}$ and a minimum of 60 $\mathrm{dB}$. The $90 \%$ percentile range $\left(5^{\text {th }}\right.$ to 95 th percentile) was $25 \mathrm{~dB}(80-105 \mathrm{~dB})$.

\section{Discussion}

High frequency tympanometry is increasingly being implemented in neonatal and infant hearing screening programmes despite a general lack of normative guidelines across age and gender. Although no large-scale $1000 \mathrm{~Hz}$ probe tone tympanometry study of infants from birth to 1 year of age has been reported the incidence of peaked tympanograms for neonatal ears in this study is similar to that of a recent report for a group of 170 babies aged between 1 and 6 days ${ }^{16}$. Using $1000 \mathrm{~Hz}$ probe tone tympanometry, a peaked tympanogram incidence of $93.4 \%$ was reported in 228 ears compared to $95 \%$ for neonates (1-4 weeks of age) in the current study. The $1000 \mathrm{~Hz}$ tympanometric peak results for neonates in the current study and those reported by Kei et al. ${ }^{16}$ are also similar to a $678 \mathrm{~Hz}$ probe tone study conducted on a group of 200 special care baby unit babies, which indicated a $91 \%$ incidence of discernible peak tympanograms ${ }^{21}$.

Double peaked tympanograms were also reported by Kei et al. ${ }^{16}$ in $1.2 \%$ of the peaked tympanogram ears compared with $6 \%$ in the current study. The higher incidence of double peaked tympanograms may be due to the larger neonatal age range in the current study (1-4 weeks) compared with that of the neonatal ears in the study by Kei et al. (1-6 days). All double peaked tympanograms in the study by Kei et al. ${ }^{16}$ were accompanied by OAE pass results, corresponding to the high percentage of ears (15/16) in the current study with OAE pass results. These results suggest that double peaked tympanograms are indicative of normal middle ear transmission and also correspond to previous reports suggesting that double peak tympanograms are not uncommon and are suggestive of normal middle ear transmission for $1000 \mathrm{~Hz}$ probe tone measurements ${ }^{2}$. 
Although present OAEs are not a perfect gold standard for the absence of middle ear pathology, their measurement is very dependent on an intact uncompromised middle ear system ${ }^{16,21}$. This reliance on normal or near normal middle ear functioning make OAE a useful measure for comparison to immittance results for providing sensitivity and specificity estimates for high frequency immittance testing in the absence of air and bone conduction tone ABR measurements. This comparison revealed a high specificity rate for both $1000 \mathrm{~Hz}$ probe tone tympanometry (95\%) and acoustic reflexes (90\%) in correctly identifying normal neonatal middle ear functioning in the current study. A significant but poorer sensitivity rate of $57 \%$ was obtained for both tympanometry and acoustic reflexes for correctly identifying middle ear pathology. The decreased sensitivity may in part be attributed to the presence of middle ear effusion in a number of ears with present but decreased OAEs ${ }^{16,21}$. Such cases result in an overlap of true positive high frequency immittance results and false negative OAE results and lead to decreased immittance sensitivity. It is for this reason that normative high frequency immittance data in the current study were compiled from neonatal ears with both an OAE pass and a tympanometric peak result.

The significantly higher static acoustic peak admittance values ( $p<0.01$; $t$ test with pooled variance) for boys in the normative sample can primarily be attributed to the difference in middle ear and tympanic membrane sizes for female and male ears ${ }^{22}$. This difference highlights the consideration of gender in establishing normative high frequency tympanometry data. Statistically significant differences in peak admittance values $(p<0.05 ; t$ test with pooled variance) for younger and older neonates indicate a general increase in admittance with increasing age. However, this trend is observed mostly for male ears. The close relationship between the 5 th percentiles grouped for gender across age indicates the significance of this value as a more robust diagnostic criterion than the more variable 95th percentile, which is not usually an indication of pathology in neonates ${ }^{17}$. Using the 5th percentile as the primary diagnostic criterion requires differentiation for normative data between only gender for all age female neonates and male neonates younger than 2 weeks of age. Male neonates 2-4 weeks of age have a significant increase in 5th percentile peak admittance values that should considered when assessing $1000 \mathrm{~Hz}$ probe tone tympanometry results.

The static peak admittance values of the current study correspond to the values reported by Margolis et al. ${ }^{17}$ for 105 NICU and 46 full-term infant ears. The 5th percentile value for newborn neonates ( 0 weeks age) in the current study was 1.2 mmho compared to 1.3 mmho for the study by Margolis et al. ${ }^{17}$ of NICU infants who were tested at a mean age of 37 weeks. Neonates in the current study aged between 1 and 4 weeks presented with a static peak admittance value of $1.5 \mathrm{mmho}$ compared to $1.2 \mathrm{mmho}$ for full-term infants in the study by Margolis et al. This difference may be accounted for by the small sample size used and the fact that the gender and race of subjects were not stated or controlled for in the latter study. The results from the present study clearly indicate that the admittance values measured with a $1000 \mathrm{~Hz}$ probe tone are significantly different between genders and the gender constitution of a sample may therefore have a significant effect on the average admittance value for a group of neonates. 
Although the importance of peak pressure values for determining pathology in infant ears is not clearly understood and further studies have been recommended, previous reports have indicated a significant relationship between middle ear pressure, using a $1000 \mathrm{~Hz}$ probe tone, and OAE failure in neonatal ears ${ }^{2,17,23}$. Although there were no statistically significant gender or age effects for tympanic peak pressure values, the 5th and 95th percentile values were consistently lower for female ears across age. The $90 \%$ range of tympanic peak pressure values for newborn neonates ( 0 weeks of age) in the current study was 140 daPa compared to $146 \mathrm{daPa}$ for the NICU infants (37 weeks gestational age) reported by Margolis et al. ${ }^{17}$. This range increased for older neonates in the current study (2-4 weeks of age) to $210 \mathrm{daPa}$ compared to $243 \mathrm{daPa}$ for the well-baby ears (2-4 weeks of age) reported by Margolis et al. ${ }^{17}$. Both studies demonstrate an increasing range of normative tympanic peak pressure values with increasing age. The slightly larger range for the older neonates reported by Margolis et al. ${ }^{17}$ compared with the current study may be attributed to fact that gender was not reported or controlled for in the study by Margolis et al. and the sample size was limited.

The incidence (86\%) of present acoustic reflexes in the current sample of neonates compares well with results from a normative study by Sprague et al. ${ }^{24}$ in which a $660 \mathrm{~Hz}$ probe tone was used with a $1000 \mathrm{~Hz}$ stimulus to measure reflexes in a sample of normal neonates. Present reflexes were measured in $81 \%$ of the subjects tested. Another study by Sutton et al. ${ }^{21}$ using a high frequency probe tone $(678 \mathrm{~Hz})$ to elicit reflexes in high-risk special care neonates, reported the presence of acoustic reflexes in only $42 \%$ of ears (71/168). This reduced incidence compared with the high incidence rate in the current study can be attributed to the risk status and young age of the neonates as well as to the use of a $678 \mathrm{~Hz}$ probe tone instead of a $1000 \mathrm{~Hz}$ probe tone. The high incidence of present reflexes recorded (86\%) in the current study compared with the poor reliability of recording present reflexes using low frequency probe tones in infants can be attributed to the following facts: (1) a $1000 \mathrm{~Hz}$ probe tone was used, (2) an ipsilateral stimulus was used, and (3) a mid-frequency $(1000 \mathrm{~Hz})$ stimulus was used to activate the reflex ${ }^{18}$.

The mean acoustic reflex threshold (93 dB) using a $1000 \mathrm{~Hz}$ probe tone and $1000 \mathrm{~Hz}$ ipsilateral stimulus for neonates in the current study was $11 \mathrm{~dB}$ higher than the same mean threshold ( $82 \mathrm{~dB}$ ) using a $220 \mathrm{~Hz}$ probe tone in adults ${ }^{25}$. A study on neonates using a $660 \mathrm{~Hz}$ probe tone to elicit ipsilateral $1000 \mathrm{~Hz}$ acoustic reflexes revealed the same 11 $\mathrm{dB}$ mean threshold difference when compared with the current study ${ }^{24}$. The $1000 \mathrm{~Hz}$ probe tone reflex thresholds for a $1000 \mathrm{~Hz}$ stimulus in normal neonates are therefore elevated by approximately $10 \mathrm{~dB}$ compared with conventional adult acoustic reflex thresholds using a $220 \mathrm{~Hz}$ probe tone.

\section{Conclusion}

Clinical experience and initial reports demonstrate that high frequency immittance measurements using a $1000 \mathrm{~Hz}$ probe tone assist in clarifying false positive screening results due to middle ear pathology or transient middle ear effusion, which is a common occurrence in neonatal hearing screening programmes. The normative criteria for interpreting $1000 \mathrm{~Hz}$ probe tone immittance results in neonates (0-4 weeks of age) 
demonstrate the important effect of age and gender on tympanograms and necessitate consideration of these variables when compiling norms. Correct identification of middle ear status in the neonatal period could direct timely and correct referrals to medical and audiological personnel that may lead to improved efficacy of neonatal hearing screening programmes.

\section{References}

1. Mencher, GT and DeVoe, SJ. (2001) Universal newborn screening: a dream realized or a nightmare in the making?. Scand Audiol 30:Suppl 53 , pp. 15-21.

2. Thornton, ARD, Kimm, L., Kennedy, CR and Cafarelli-Dees, D. (1993) Externaland middle-ear factors affecting evoked otoacoustic emissions in neonates. $\mathrm{Br} \mathrm{J}$ Audiol 27, pp. 319-327.

3. Keefe, DH, Zhao, F., Neely, ST, Gorga, MP and Vohr, BR. (2003) Ear-canal acoustic admittance and reflectance effects in human neonates. I. Predictions of otoacoustic emission and auditory brainstem responses. J Acoust Soc Am 113, pp. 389-406.

4. Engel, J., Mahler, E., Anteunis, L., Marres, E. and Zielhuis, G. (2001) Why are NICU infants at risk for chronic otitis media with effusion?. Int J Pediatr Otorhinolaryngol 57, pp. 137-144.

5. Boone, RT, Bower, CM and Martin, PF. (2005) Failed newborn hearing screens as presentation for otitis media with effusion in the newborn population. Int J Pediatr Otorhinolaryngol 69, pp. 393-397.

6. Hall, JW III, Smith, SD and Popelka, GR. (2004) Newborn hearing screening with combined otoacoustic emissions and auditory brainstem responses. J Am Acad Audiol 15, pp. 414-425.

7. Yeo, SW, Park, SN, Park, YS and Suh, BD. (2002) Effect of middle-ear effusion on otoacoustic emissions. J Laryngol Otol 116, pp. 794-799.

8. Koivun, P., Uhari, M., Laitakari, K., Alho, OP and Luotonen, J. (2000)

Otoacoustic emissions and tympanometry in children with otitis media. Ear Hear 21, pp. 212-217.

9. El-Refaie, A., Parker, DJ and Bamford, JM. (1996) Otoacoustic emission versus ABR screening: the effect of external and middle ear abnormalities in a group of SCBU neonates. Br J Audiol 30, pp. 3-8.

10. Taylor, CL and Brooks, RP. (2000) Screening for hearing loss and middle-ear disorders in children using TEOAEs. Am J Audiol 9, pp. 50-55. 
11. Holte, L., Margolis, RH and Cavanaugh, R. (1991) Developmental changes in multifrequency tympanograms. Audiology 30, pp. 1-24.

12. Hunter, LL and Margolis, RH. (1992) Multifrequency tympanometry: current clinical application. Am J Audiol 1, pp. 33-43.

13. McKinley, AM, Grose, JH and Roush, J. (1997) Multifrequency tympanometry and evoked acoustic emissions in neonates during the first 24 hours of life. J Am Acad Audiol 8, pp. 218-223.

14. Meyer, SE, Jardine, CA and Deverson, W. (1997) Developmental changes in tympanometry: a case study. Br J Audiol 31, pp. 189-195.

15. Keefe, DH and Levi, E. (1996) Maturation of the middle and external ears: acoustic power-based responses and reflectance tympanometry. Ear Hear 17, pp. 361-373.

16. Kei, J., Allison-Levick, J., Dockray, J., Harrys, R., Kirkegard, C. and Wong, J. (2003) High-frequency $(1000 \mathrm{~Hz})$ tympanometry in normal neonates. J Am Acad Audiol 14, pp. 21-28.

17. Margolis, RH, Bass-Ringdahl, S., Hanks, WD, Holte, K. and Zapala, DA. (2003) Tympanometry in newborn infants - $1 \mathrm{KHz}$ norms. J Am Acad Audiol 14, pp. 383-392.

18. Purdy, SC and Williams, MJ. (2000) High frequency tympanometry: a valid and reliable immittance test protocol for young infants? New Zealand Audiological Society Bulletin 10, pp. 9-24.

19. Olusanya, BO, Luxon, LM and Wirz, SL. (2004) Benefits and challenges of newborn hearing screening for developing countries. Int J Pediatr Otorhinolaryngol 68, pp. 287-305.

20. Van Cauwenberge, PB, Vinck, B., De Vel, E. and Dhooge, I. (Lim, DJ, Bluestone, CD, Casselbrandt, M., Klein, JO and Ogra, PL eds.) (1996) Tympanometry and click evoked otoacoustic emissions in secretory otitis media: are C-EOAEs consistently absent in B-tympanograms? Recent advances in otitis media pp. 139-141. BC Decker, Ontario

21. Sutton, GJ, Gleadle, P. and Rowe, SJ. (1996) Tympanometry and otoacoustic emissions in a cohort of special care neonates. Br J Audiol 30, pp. 9-17.

22. Palmu, A., Puhakka, H., Rahko, T. and Takala, AK. (1999) Diagnostic value of tympanometry in infants in clinical practice. Int J Pediatr Otorhinolaryngol 49, pp. 207-213. 
23. Owens, JJ, McCoy, MJ, Lonsbury-Martin, BC and Martin, GK. (1992) Influence of otitis media on evoked otoacoustic emissions in children. Semin Hear 13, pp. 5356.

24. Sprague, BH, Wiley, TL and Goldstein, R. (1985) Tympanometric and acousticreflex studies in neonates. J Speech Hear Res 28, pp. 265-272.

25. Wiley, TL, Oviat, DL and Block, MG. (1987) Acoustic immittance measures in normal ears. J Speech Hear Res 330, pp. 161-170. 